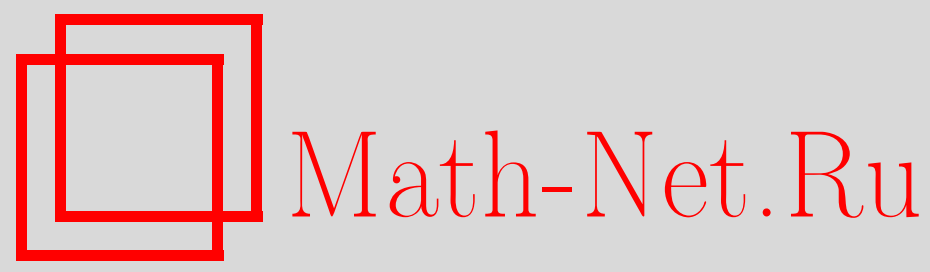

Х. К. Гарсиа, О структуре конуса нормальных неограниченных вполне положительных отображений, Матем. заметки, 1999, том 65, выпуск 2, 194-205

DOI: https://doi.org/10.4213/mzm1041

Использование Общероссийского математического портала Math-Net.Ru подразумевает, что вы прочитали и согласны с пользовательским соглашением http://www.mathnet.ru/rus/agreement

Параметры загрузки:

IP: 35.173 .219 .12

26 апреля 2023 г., 12:21:20 


\title{
О СТРУКТУРЕ КОНУСА НОРМАЛЬНЫХ НЕОГРАНИЧЕННЫХ ВПОЛНЕ ПОЛОЖИТЕЛЬНЫХ ОТОБРАЖЕНИЙ
}

\author{
Хулио С. Гарсиа
}

\begin{abstract}
В статье предлагается конструктивная характеризация неограниченных вполне положительных отображений, которые А. М. Чеботарев использовал ранее в теории квантовых динамических полугрупп. Доказано, что такие конусы порождаются положительным самосопряженным "весовым" оператором $\Lambda \in \mathscr{B}(\mathscr{H})$ : для любого вполне положительного нормального ограниченного отображения $\Phi(\cdot) \in C P n_{*}(\mathscr{F})$ существует вполне положительное нормальное ограниченное отображение $R \in C P n(\mathscr{H})$ такое, что $\Phi(\cdot)=\Lambda R(\cdot) \Lambda$. Данный класс содержит отображения, являющиеся незамыкаемьми полуторалинейными формами.

Библиограффия: 12 названий.
\end{abstract}

1. Введение. В работе изучается структура специального класса конусов неограниченных вполне положительных отображений, обозначенных через $C P n_{*}(\mathscr{F})$, которые были введены А. М. Чеботаревьм [1], [2]. Элементами таких конусов являются вполне положительные операторные коэффициенты формальных инфинитезимальных генераторов широкого класса квантовых динамических полугрупп. Данные исследования были начаты в работе [3].

Пусть $\mathscr{H}$ - сепарабельное гильбертово пространство, называемое исходныл, снабженное скалярным произведением $\langle\cdot, \cdot\rangle$ и нормой $\|\cdot\|$. Пусть $\mathscr{B}(\mathscr{H})$ - алгебра всех ограниченных линейных операторов на $\mathscr{H}$. Пусть $\mathscr{F}$ - топологическое векторное пространство, плотно и непрерьвно вложенное в $\mathscr{H}$.

Данная работа состоит из четырех пунктов. В п. 2, где $\mathscr{F} \subset \mathscr{H}$ считается гильбертовым пространством, в теореме 1 и следствии 1 дана конструктивная характеризация конуса $C P n_{*}(\mathscr{F})$, аналогичная теореме Крауза. В п. 3 пространство $\mathscr{F}$ отождествляется с проективным пределом гильбертовых пространств, а характеризация соответствующего конуса $C P n_{*}(\mathscr{F})$ содержится в теореме 2 и следствии 2 . В п. 4 рассмотрены некоторые примеры и приведены комментарии к ним.

Напомним определения конусов $C P n(\mathscr{H})$ и $C P n_{*}(\mathscr{F})$ и приведем их основные свойства.

ОПРЕДЕЛЕНИЕ 1. $C P n(\mathscr{H})$ есть конус всех вполне положительных нормальных линейных отображений (супероператоров) $R: \mathscr{B}(\mathscr{H}) \rightarrow \mathscr{B}(\mathscr{H})$ (см. [4], [5]).

Работа выполнена при частичной финансовой поддержке фонда CONACYT (Мексика), грант № 0233P-E9506. 
Пусть $R \in C P n(\mathscr{H})$. Это отображение определено всюду на банаховой алгебре $\mathscr{B}(\mathscr{H})$. Можно доказать, что нормальность отображения $R$ влечет его замкнутость, так что $R$ является ограниченным.

Теорема Крауза [6, теорема 3.3, с. 217] дает конструктивную характеризацию конуса $C P n(\mathscr{H})$, исчерпьвающую все возможные примеры отображений из $C P n(\mathscr{H})$. А именно, $R \in C P n(\mathscr{H})$ тогда и только тогда, когда существует последовательность операторов $\left\{A_{k}\right\}$ из $\mathscr{B}(\mathscr{H})$ такая, что $\sum_{k} A_{k}^{*} A_{k}$ сильно сходится к некоторому ограниченному оператору и

$$
R(X)=\sum_{k} A_{k}^{*} X A_{k}, \quad X \in \mathscr{B}(\mathscr{H}) .
$$

Интерес к теории неограниченных вполне положительных отображений основывается на том, что они являются коэффициентами линдбладовской формы инфинитезимальных генераторов для широкого класса квантовьг динамических полугрупп. Предположив, что $\mathscr{H}=L_{2}(\mathbb{R})$, в качестве примера можно привести формальный генератор

$$
\mathscr{L}(B)=\Phi(B)-\frac{1}{2}(\Phi(I) B+B \Phi(I))+i[H, B]
$$

где $B \in \mathscr{B}(\mathscr{H}), \Phi(B)=-\frac{1}{2} \partial_{x} B \partial_{x}$ и $H=0$. Этот генератор можно рассматривать как квантовое расширение лапласиана в $\mathbb{R}$, при этом соответствующее ему марковское эволюционное уравнение (master equation) является квантовым расширением уравнения теплопроводности. Отображение $\Phi(\cdot)$ представляет собой естественный пример вполне положительного неограниченного отображения.

Введем на $C P n(\mathscr{H})$ семейство псевдометрик

$$
\mathscr{R}_{\mathscr{F}}=\left\{\Theta_{\mathbb{A}, \mathbb{B}}: \mathbb{A} \subset \mathscr{F}, \mathbb{B} \subset \mathscr{B}(\mathscr{H})\right\}
$$

индексированное парами $(\mathbb{A}, \mathbb{B})$, где $\mathbb{A}$ - абсолютно вьпуклое компактное множество в $\mathscr{F}, \mathbb{B}$ - абсолютно выпуклое компактное множество относительно ультраслабой топологии в $\mathscr{B}(\mathscr{H})$ и

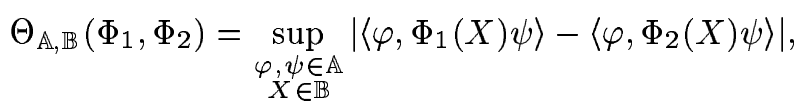

где $\Phi_{1}, \Phi_{2} \in C P n(\mathscr{H})$. Отметим, что семейство псевдометрик (1) зависит от пространства $\mathscr{F}$.

Напомним, что предкомпактные множества в ультраслабой топологии в $\mathscr{B}(\mathscr{H})$ являются ограниченными по норме [5]. Семейство $\mathscr{R} \mathscr{F}$ индуцирует в $C P n(\mathscr{H})$ структуру равномерного топологического пространства. Следовательно, существует пополнение [7, теорема VI.28, с. 196].

ОПРЕДЕЛЕНИЕ $2 . C P n_{*}(\mathscr{F})$ представляет собой пополнение $C P n(\mathscr{H})$ относительно псевдометрики (1).

В дальнейшем для псевдометрики в $C P n_{*}(\mathscr{F})$ мы используем те же обозначения, что и в (1). 
Из конструкции пополнений равномерных топологических пространств [7, теорема VI.28, с. 196] следует, что $C P n_{*}(\mathscr{F})$ является конусом всех отображений $\Phi: \mathscr{B}(\mathscr{H}) \times$ $\mathscr{F} \times \mathscr{F} \rightarrow \mathbb{C}$ таких, что существует сеть $\left\{\Phi_{\alpha}\right\}_{\alpha \in \mathscr{I}}(\mathscr{I}-$ упорядоченное множество) в $C P n(\mathscr{H})$, сходящаяся к $\Phi$ в следующем смысле: для всякого $\mathscr{F}$-компактного подмножества $\mathbb{A} \subset \mathscr{F}$ и всех ограниченных по норме подмножеств $\mathbb{B} \subset \mathscr{B}(\mathscr{H})$

$$
\Theta_{\mathbb{A}, \mathbb{B}}\left(\Phi_{\alpha}, \Phi\right) \rightarrow 0
$$

В случае $\mathscr{F}=\mathscr{H}$ очевидно, что $C P n_{*}(\mathscr{H})=C P n(\mathscr{H})$. Если пространство $\mathscr{F}$ метризуемо, то следующие свойства являются необходимьми условиями того, что объект $\Phi \in C P n_{*}(\mathscr{F})$.

Алгебраические свойства:

a) $\Phi(\cdot)[\cdot, \cdot]$ - полуторалинейное отображение относительно векторных переменных (находящихся в $[\cdot, \cdot])$ и линейным отображением относительно операторной переменной (находящейся в $(\cdot))$;

б) $\Phi(\cdot)[\cdot, \cdot]$ - вполне положительное отображение, т.е.

$$
\sum_{i, j=1}^{n} \Phi\left(X_{i}^{*} X_{j}\right)\left[\psi_{i}, \psi_{j}\right] \geqslant 0 \quad \text { для } n \in \mathbb{N}, \quad \psi_{i} \in \mathscr{F}, \quad X_{i} \in \mathscr{B}(\mathscr{H}) .
$$

\section{Свойства непрерывности:}

в) при фиксированном $X \in \mathscr{B}(\mathscr{H})$ отображение $\Phi(X)[\cdot, \cdot]$ непрерьвно в $\mathscr{F}$;

г) при фиксированном $\psi \in \mathscr{F}$ отображение $\Phi(\cdot)[\psi, \psi]$ является нормальным линейным функционалом на $\mathscr{B}(\mathscr{H})$, т.е.

$$
X=\sup _{n} X_{n} \Longrightarrow \sup _{n} \Phi\left(X_{n}\right)[\psi, \psi]=\Phi(X)[\psi, \psi]
$$

для любой возрастающей ограниченной по норме последовательности $\left\{X_{n}\right\}$ положительных элементов из $\mathscr{B}(\mathscr{H})$.

В действительности алгебраические свойства а) и б) являются следствием поточечной сходимости элементов $C P n(\mathscr{H})$, а свойства непрерывности в) и г) следуют из равномерной сходимости на компактных множествах.

Если $R \in C P n(\mathscr{H})$, то оно отображает положительные операторы в положительные. Так как для любого ограниченного самосопряженного оператора $X$ справедливо неравенство $X \leqslant\|X\| I$, то

$$
\|R(X)\| \leqslant\|X\| \cdot\|R(I)\| .
$$

Аналогичное неравенство имеет место для элементов $C P n_{*}(\mathscr{F})$, как следствие алгебраических свойств а) и б):

$$
|\Phi(X)[\psi, \psi]| \leqslant\|X\| \cdot|\Phi(I)[\psi, \psi]|,
$$

где оператор $X$ ограничен и самосопряжен, а $\psi \in \mathscr{F}$. В дальнейшем будем предполагать считать пространство $\mathscr{F}$ метризуемым.

Естественно предположить, что свойства а)-г) являются также достаточными условиями того, что элемент $\Phi \in C P n_{*}(\mathscr{F})$. Докажем это предположение в случаях, когда $\mathscr{F}$ представляет собой гильбертово пространство (п. 2) и проективньй предел гильбертовых пространств (п. 3). В последнем случае примерами могут служить $\mathscr{H}=L_{2}(\mathbb{R})$ и 
$\mathscr{F}$ - пространство Шварца быстро убьвающих гладких функций. Известно [8], что пространство Шварца является проективньм пределом пространств $\left\{\mathscr{H}_{k}\right\}_{k \geqslant 0}$, где $\mathscr{H}_{k}-$ область определения $N^{k / 2}$, а $N$ - так назьваемьй гамильтониан гармонического осииллятора

$$
N=\frac{1}{2}\left(-\frac{d^{2}}{d x^{2}}+x^{2}+1\right) .
$$

Более общие реализации пространства $\mathscr{F}$, а также другие примеры будут рассмотрены в последующих работах.

Обозначения и предположения. В настоящей работе мы рассматриваем комплексные сепарабельные гильбертовы пространства, полуторалинейные формы предполагаем антилинейными по левому и линейными по правому аргументам; для полуторалинейной формы $a$ используем запись $a[\varphi, \psi]$ и для соответствуюшей квадратичной формы $a[\psi]=a[\psi, \psi][9]$. Через $\mathbb{B}_{*}(\mathscr{F})$ обозначаем пространство совместно непрерывных полуторалинейных форм на $\mathscr{F}$.

В этих обозначениях, поскольку свойства а)-г) являются необходимыми условиями, мы утверждаем, что множество $C P n_{*}(\mathscr{F})$ содержится в множестве всех вполне положительных нормальных в смысле б) и г) линейных отображений $\Phi: \mathscr{B}(\mathscr{H}) \rightarrow \mathbb{B}_{*}(\mathscr{F})$.

В случае, когда $\mathscr{F}$ является гильбертовым пространством, мы отождествляем $\mathbb{B}_{*}(\mathscr{F})$ с пространством $\mathbb{B}(\mathscr{F})$ ограниченных операторов на $\mathscr{F}$ с помощью равенства

$$
a[\varphi, \psi]=\langle\varphi, A \psi\rangle_{\mathscr{F}}
$$

где $\langle\cdot, \cdot\rangle_{\mathscr{F}}-$ скалярное произведение в $\mathscr{F}, a \in \mathbb{B}_{*}(\mathscr{F})$ и $A-$ элемент $\mathbb{B}(\mathscr{F})$, соответствуюший полуторалинейной форме $a$.

2. Пополнения, порожденные гильбертовыми пространствами. Рассмотрим простейший случай, когда $\mathscr{F}$ представляет собой гильбертово пространство, плотным непрерьвньм образом вложенное в $\mathscr{H}$. Прежде всего установим одно простое свойство конусов $C P n_{*}(\mathscr{F})$ для метризуемых топологических векторных пространств $\mathscr{F}$ общего вида.

ПРЕДЛОЖЕНИЕ 1. Пусть $\mathscr{F}_{1}$ и $\mathscr{F}_{2}$ - метризуемые векторные пространства, а $\mathscr{H}$ - гильбертово пространство, $\mathscr{F}_{1}$ плотно и непрерывно вложсено в $\mathscr{F}_{2}$, а $\mathscr{F}_{2}$ плотно и непрерывно влохено в $\mathscr{H}$. Тогда

$$
C P n_{*}\left(\mathscr{F}_{2}\right) \subseteq C P n_{*}\left(\mathscr{F}_{1}\right)
$$

Отображение вложения является равномерно непрерывным.

ДокАЗАТЕЛЬСтво. Естественно рассматривать $\mathbb{B}_{*}\left(\mathscr{F}_{2}\right)$ как подпространство в $\mathbb{B}_{*}\left(\mathscr{F}_{1}\right)$. Действительно, так как $\mathscr{F}_{1}$ содержится в $\mathscr{F}_{2}$, всякая полуторалинейная форма на $\mathscr{F}_{2}$ является также полуторалинейной формой на $\mathscr{F}_{1}$. Поскольку топология $\mathscr{F}_{1}$ сильнее топологии, порожденной $\mathscr{F}_{2}$, это сужение является непрерьвной полуторалинейной формой на $\mathscr{F}_{1}$. Следовательно, $\mathbb{B}_{*}\left(\mathscr{F}_{2}\right) \subseteq \mathbb{B}_{*}\left(\mathscr{F}_{1}\right)$.

Отметим, что любое компактное множество в $\mathscr{F}_{1}$ является также компактным множеством в $\mathscr{F}_{2}$. Отсюда $\mathscr{R}_{\mathscr{F}_{1}} \subseteq \mathscr{R}_{\mathscr{F}_{2}}$. Это доказывает, что тождественное отображение

$$
i:\left(C P n(\mathscr{H}), \mathscr{R}_{\mathscr{F}_{2}}\right) \rightarrow\left(C P n(\mathscr{H}), \mathscr{R}_{\mathscr{F}_{1}}\right)
$$


равномерно непрерьвно. Далее, существует единственное равномерно непрерьвное расширение отображения $i$, действующее из одного пополнения в другое:

$$
\tilde{\imath}: C P n_{*}\left(\mathscr{F}_{2}\right) \rightarrow C P n_{*}\left(\mathscr{F}_{1}\right) .
$$

Рассмотрим $\Phi \in C P n_{*}\left(\mathscr{F}_{2}\right)$. Тогда $\Phi(X) \in \mathbb{B}_{*}\left(\mathscr{F}_{2}\right)$ для любого $X \in \mathscr{B}(\mathscr{H})$. Таким образом, $\Phi(X) \in \mathbb{B}_{*}\left(\mathscr{F}_{1}\right)$ как сужение на $\mathscr{F}_{1} ;$ кроме того, в $C P n(\mathscr{H})$ существует сеть $\left\{\Phi_{\alpha}\right\}_{\alpha}$, сходящаяся к $\Phi$ в топологии, порожденной $\mathscr{R}_{\mathscr{F}_{2}}$. В свою очередь, $\Phi_{\alpha}=i\left(\Phi_{\alpha}\right)$ сходится к $\tilde{\imath}(\Phi)$ в топологии, порожденной $\mathscr{R}_{\mathscr{F}_{1}}$. Следовательно, $[\tilde{\imath}(\Phi)](X)$ является сужением $\Phi(X)$ на $\mathscr{F}_{1}$, и включение $\tilde{\imath}$ следует из плотности $\mathscr{F}_{1}$ в $\mathscr{F}_{2}$. Таким образом, получаем (6).

Далее мы не будем использовать обозначение $\tilde{\imath}$, называя его отображением вложения. Частичная упорядоченность по отношению к $\mathscr{F}_{1}$ и $\mathscr{F}_{2}$ очевидна.

ПРЕДЛОЖЕНИЕ 2. Существует плотно определенный положительный самосопряженный оператор $\Lambda$ в $\mathscr{H}$, удовлетворяющий условиям

$$
\mathscr{F}=\operatorname{dom} \Lambda \quad(\text { как множества }), \quad\langle\Lambda \psi, \Lambda \varphi\rangle=\langle\psi, \varphi\rangle_{\mathscr{F}},
$$

а 0 принадлежит резольвентному множеству $\Lambda$.

ДокАЗАТЕльСтво. Поскольку пространство $\mathscr{F}$ непрерьвно вложено в $\mathscr{H}$, существует положительная константа $K$ такая, что

$$
\|u\| \leqslant K\|u\|_{\mathscr{F}}, \quad u \in \mathscr{F} .
$$

Пусть $a[\psi, \varphi]=\langle\psi, \varphi\rangle_{\mathscr{F}}$. Нетрудно показать, что квадратичная форма $a$ плотно определена, положительна и замкнута на области определения $\mathscr{F}$. Согласно первой и второй формулировкам теоремы Като [9, теоремы VI.2.1 и VI.2.23, с. 322, 331] существует плотно определенный положительный замкнутьй оператор $A$ такой, что $\operatorname{dom} A$ является плотным подпространством в $\mathscr{F}, \operatorname{dom} A^{1 / 2}=\mathscr{F}$,

$$
\begin{array}{lll}
a[\psi, \varphi]=\left\langle A^{1 / 2} \psi, A^{1 / 2} \varphi\right\rangle, & & \psi, \varphi \in \mathscr{F}, \\
a[\psi, \varphi]=\langle\psi, A \varphi\rangle, & & \varphi \in \operatorname{dom} A, \quad \psi \in \mathscr{F} .
\end{array}
$$

Пусть $\Lambda=A^{1 / 2}$. Нужно доказать, что 0 принадлежит резольвентному множеству. Для этого достаточно показать, что оператор $A$ имеет ограниченньй обратньй. Для $u \in \operatorname{dom} A$ имеем

$$
\|A u\| \cdot\|u\| \geqslant\langle u, A u\rangle=\|u\|_{\mathscr{F}}^{2} \geqslant K^{-2}\|u\|^{2} .
$$

Отсюда ясно, что ker $A=0$ и при $u \neq 0$

$$
\|A u\| \geqslant K^{-2}\|u\| .
$$

Так как оператор $A$ самосопряжен, a $\operatorname{ker} A=0$, то его область значений плотна в $\mathscr{H}$. Докажем, что множество значений range $A$ замкнуто.

Пусть $\left\{x_{n}\right\}$ - последовательность в $\operatorname{dom} A$, а $y \in \mathscr{H}$ такой, что $A x_{n} \rightarrow y$. Согласно (8) $\left\{x_{n}\right\}$ является ограниченной последовательностью в $\mathscr{H}$, из которой можно выделить подпоследовательность $\left\{y_{m}\right\}$, слабо сходящуюся к некоторому $v \in \mathscr{H}$. Для всякого $z \in \operatorname{dom} A$ имеем

$$
\langle v, A z\rangle=\lim _{m}\left\langle y_{m}, A z\right\rangle=\lim _{m}\left\langle A y_{m}, z\right\rangle=\langle y, z\rangle .
$$

Следовательно, $v \in \operatorname{dom} A^{*}=\operatorname{dom} A$ и $y=A^{*} v=A v \in \operatorname{range} A$. Так как range $A=\mathscr{H}$, то $A^{-1}$ является всюду определенным симметричным, а значит, и ограниченным оператором. 
ЗАмЕчАниЕ 1 . Поскольку $\{0\}$ принадлежит резольвентному множеству $\Lambda$, из $(7)$ следует, что оператор $\Lambda$ представляет собой унитарньй изоморфизм между $\mathscr{F}$ и $\mathscr{H}$. Этот факт является ключевым для полной характеризации $C P n_{*}(\mathscr{F})$.

ЗАмечание 2. Пусть отображение $\lambda: \mathscr{B}(\mathscr{H}) \rightarrow \mathscr{B}(\mathscr{F})$ определено равенством $\lambda(X)=\Lambda^{-1} X \Lambda$. Из унитарности $\Lambda$ как отображения из $\mathscr{F}$ в $\mathscr{H}$ следует, что $\lambda$ есть *-унитальный изоморфизм. В частности, $\lambda$ является обратимым вполне положительным нормальным отображением, сохраняюшим норму:

$$
\|\lambda(X)\|_{\mathscr{B}(\mathscr{F})}=\|X\|_{\mathscr{B}(\mathscr{H})}
$$

Обратное отображение $\lambda^{-1}$ обладает точно такими же свойствами.

ЗАмечАниЕ 3 . Согласно (8) оператор $A^{-1}$ имеет норму, не превосходящую $K^{-2}$. Из самосопряженности $A$ следует, что $\Lambda$ не меньше константы $K$, умноженной на единичньй оператор в $\mathscr{H}$. Вводя в случае необходимости новое скалярное произведение в $\mathscr{F}$, эквивалентное прежнему:

$$
\langle\langle u, v\rangle\rangle=\langle u, v\rangle_{\mathscr{F}}+\langle u, v\rangle,
$$

без потери общности можем предположить, что $K=1$ и $\Lambda \geqslant I$.

Положим $\Lambda_{\varepsilon}=\Lambda(1+\varepsilon \Lambda)^{-1}$ и $x_{\varepsilon}=(1+\varepsilon \Lambda)^{-1} x$ для $x \in \mathscr{H}$. Для $u, v, x \in \mathscr{H}$ обозначим через $|u\rangle\langle v|$ оператор единичного ранга в $\mathscr{H}$, так что $|u\rangle\langle v| x=\langle v, x\rangle u$. Для операторов с конечньм следом введем обозначение следа tr.

ПРЕДЛОЖЕНИЕ 3. Для любого подмножсества $\mathbb{A} \subset \mathscr{F}$, являющегося компактным множеством в топологии $\mathscr{F}$, сходимость

$$
\left|\Lambda x_{\varepsilon}\right\rangle\left\langle\Lambda x_{\varepsilon}|\rightarrow| \Lambda x\right\rangle\langle\Lambda x|
$$

при $\varepsilon \rightarrow 0$ является равномерной относительно нормы $\|\cdot\|_{\operatorname{tr}}$ для каждого $x \in \mathbb{A}$.

ДокАЗАТЕЛЬСТво. Пусть $z=\Lambda x$. Тогда $z_{\varepsilon}=\Lambda x_{\varepsilon}$ и согласно неравенству треугольника

$$
\|\left|z_{\varepsilon}\right\rangle\left\langle z_{\varepsilon}|-| z\right\rangle\langle z|\left\|_{\mathrm{tr}} \leqslant 2\right\| z_{\varepsilon}-z\|\cdot\| z\|=2\| \Lambda x_{\varepsilon}-\Lambda x\|\cdot\| \Lambda x \|
$$

а это выражение стремится к 0 при $\varepsilon \rightarrow 0$. Данная сходимость равномерна для $x \in \mathbb{A}$, так как $(1+\varepsilon \Lambda)^{-1}$ возрастает до 1 и $\Lambda x$ принадлежит множеству $\Lambda(\mathbb{A}) \subset \mathscr{H}$, которое является компактньм в силу непрерывности $\Lambda$ как оператора, действующего из $\mathscr{F}$ в $\mathscr{H}$. Таким образом, $\left\|\left(I-(1+\varepsilon \Lambda)^{-1}\right) u\right\|$ стремится к 0 равномерно при $\varepsilon \rightarrow 0$ для $u \in \Lambda(\mathbb{A})$ в силу теоремы Дини.

Следующий результат дает полную характеризацию конуса $C P n_{*}(\mathscr{F})$.

Tеорема 1. Пусть $\mathscr{G}=\{\lambda \circ R: R \in C P n(\mathscr{H})\}$, а $\mathscr{E}-$ множсество всех әлементов $\Phi$, удовлетворяющих условиям а) - г). Тогда $\mathscr{G}=C P n_{*}(\mathscr{F})=\mathscr{E}$. 
ДокАЗАтЕльство. Покажем, что $\mathscr{G} \subset C P n_{*}(\mathscr{F})$ и $\mathscr{E} \subset \mathscr{G}$. Тогда для завершения доказательства достаточно убедиться, что $C P n_{*}(\mathscr{F}) \subset \mathscr{E}$.

Пусть $R \in C P n(\mathscr{H})$. Произвольньй элемент вида $\lambda \circ R$ является отображением из $\mathscr{B}(\mathscr{H})$ в $\mathscr{B}(\mathscr{F})=\mathbb{B}_{*}(\mathscr{F})$ (обозначения см. в п. 1$)$. При любом $X \in \mathscr{B}(\mathscr{H})(\lambda \circ R)(X)$ имеет точное выражение в виде полуторалинейной формы:

$$
(\lambda \circ R)(X)[\varphi, \psi]=\left\langle\varphi, \Lambda^{-1} R(X) \Lambda \psi\right\rangle_{\mathscr{F}}=\langle\Lambda \varphi, R(X) \Lambda \psi\rangle, \quad \varphi, \psi \in \mathscr{F} .
$$

Положим $R_{\varepsilon}(X)=\Lambda_{\varepsilon} R(X) \Lambda_{\varepsilon}$. Заметим, что $R_{\varepsilon}(\cdot) \in C P n(\mathscr{H})$, так как $\Lambda_{\varepsilon} \in \mathscr{B}(\mathscr{H})$. Необходимо доказать, что $R_{\varepsilon} \rightarrow \lambda \circ R$ при $\varepsilon \rightarrow 0$ относительно псевдометрики (2). Действительно, используя (3), (9) и самосопряженность $\Lambda_{\varepsilon}$, для $\psi=\varphi \in \mathscr{F}$ имеем

$$
\begin{aligned}
\left|\left\langle\psi, R_{\varepsilon}(X) \psi\right\rangle-(\lambda \circ R)(X)[\psi]\right| & =\left|\left\langle\Lambda \psi_{\varepsilon}, R(X) \Lambda \psi_{\varepsilon}\right\rangle-\langle\Lambda \psi, R(X) \Lambda \psi\rangle\right| \\
& =\left|\operatorname{tr}\left(R(X)\left[\left|\Lambda \psi_{\varepsilon}\right\rangle\left\langle\Lambda \psi_{\varepsilon}|-| \Lambda \psi\right\rangle\langle\Lambda \psi|\right]\right)\right| \\
& \leqslant\|X\| \cdot\|R(I)\| \cdot \|\left|\Lambda \psi_{\varepsilon}\right\rangle\left\langle\Lambda \psi_{\varepsilon}|-| \Lambda \psi\right\rangle\langle\Lambda \psi| \|_{\text {tr }} .
\end{aligned}
$$

Это неравенство и предложение 3 показьвают, что сходимость равномерна, если $X$ является сужением на ограниченное по норме подмножество $\mathscr{B}(\mathscr{H})$ и $\psi$ принадлежит некоторому компактному подмножеству $\mathscr{F}$. Таким образом, $\lambda \circ R \in C P n_{*}(\mathscr{F})$.

С другой стороны, пусть $\Phi \in \mathscr{E}$. При каждом $X \in \mathscr{B}(\mathscr{H}) \quad \Phi(X) \in \mathbb{B}_{*}(\mathscr{F})=\mathbb{B}(\mathscr{F})$ вполне положительно и нормально в смысле условий б), в). Пусть $R=\lambda^{-1}$ ○ $\Phi$. Тогда $R$ представляет собой линейное отображение из $\mathscr{B}(\mathscr{H})$ в себя. Вполне положительность и нормальность $R$ следуют из его представления в виде суперпозиции вполне положительного и нормального отображений. Следовательно, $R \in C P n(\mathscr{H})$ и $\Phi=\lambda \circ R \in \mathscr{G}$.

СледСТВИЕ 1. 1) Для любого $\Phi \in C P n_{*}(\mathscr{F})$, существует последовательность $\left\{A_{k}\right\} \in \mathscr{B}(\mathscr{H})$ ограниченных операторов в $\mathscr{H}$ такая, что $\sum_{k} A_{k}^{*} A_{k}$ сильно сходumcr $u$

$$
\Phi(X)[\psi, \varphi]=\sum_{k}\left\langle A_{k} \Lambda \psi, X A_{k} \Lambda \varphi\right\rangle, \quad X \in \mathscr{B}(\mathscr{H}), \quad \psi, \varphi \in \mathscr{H} .
$$

2) Свойства а)-г) являются достаточными условиями того, что әлемент $\Phi \in C P n_{*}(\mathscr{F})$.

3) Произвольный әлемент из $C P n_{*}(\mathscr{F})$ является последовательностью әлементов из $C P n(\mathscr{H})$ в топологии, индуиированной $\mathscr{R}_{\mathscr{F}}$.

ДокАЗАтЕльство. Пусть $R \in C P n(\mathscr{H})$ и $\Phi=\lambda \circ R$. Для доказательства утверждения 1) достаточно напомнить, что согласно теореме Крауза существует последовательность $\left\{A_{k}\right\}$ в $\mathscr{B}(\mathscr{H})$ такая, что $\sum_{k} A_{k}^{*} A_{k}$ сильно сходится и

$$
R(X)=\sum_{k} A_{k}^{*} X A_{k}, \quad X \in \mathscr{B}(\mathscr{H}) .
$$

Следовательно, согласно (9) имеем

$$
\Phi(X)[\varphi, \psi]=\langle\Lambda \varphi, R(X) \Lambda \psi\rangle=\sum_{k}\left\langle A_{k} \Lambda \varphi, X A_{k} \Lambda \psi\right\rangle .
$$

Отсюда непосредственно следует утверждение 2). Утверждение 3) доказывается на основе теоремы 1. А именно, возьмем $\varepsilon=1 / n$. Тогда $\left\{R_{1 / n}\right\}_{n \in \mathbb{N}}$ является требуемой аптроксимирующей последовательностью. 
3. Пополнения, порожденные проективными пределами. Предположим, что $\mathscr{F}$ - счетное гильбертово пространство, т.е. полное локально выпуклое векторное пространство с топологией, заданной последовательностью гильбертовых полунорм $\left\{\|\cdot\|_{n}\right\}_{n \in \mathbb{N} \cup\{0\}} \cdot$ Пусть $\mathscr{H}_{n}$ - пополнение $\mathscr{F}$ относительно полунормы $\|\cdot\|_{n}$. Известно, что $\mathscr{F}$ является проективным пределом последовательности гильбертовых пространств $\mathscr{H}_{n}$ и, следовательно, представляет собой рефлексивноепространство Фреше [8, утверждение 1.2.1, с. 4], что, в частности, означает его метризуемость.

Предположим, что пространства $\mathscr{H}_{n}$ образуют убывающую последовательность

$$
\mathscr{H}_{0} \supseteq \mathscr{H}_{1} \supseteq \cdots \supseteq \mathscr{F}, \quad \mathscr{F}=\bigcap_{n} \mathscr{H}_{n}
$$

и

$$
\|u\|_{n+1} \leqslant\|u\|_{n}, \quad u \in \mathscr{F} .
$$

Так как пространство $\mathscr{F}$ плотно в каждом $\mathscr{H}_{n}$, то $\mathscr{H}_{n+1}$ плотным непрерывным образом вложено в $\mathscr{H}_{n}$.

Примем $\mathscr{H}_{0}$ в качестве исходного гильбертова пространства $\mathscr{H}$. Согласно предложению 1 существует возрастающая последовательность

$$
C P n(\mathscr{H}) \subseteq C P n_{*}\left(\mathscr{H}_{1}\right) \subseteq C P n_{*}\left(\mathscr{H}_{2}\right) \subseteq \cdots \subseteq C P n_{*}(\mathscr{F})
$$

ТЕОрема 2. Имеет место представление

$$
C P n_{*}(\mathscr{F})=\bigcup_{k} C P n_{*}\left(\mathscr{H}_{k}\right) .
$$

ДокАЗАТЕЛЬСтво. Согласно (12) объединение множеств $C P n_{*}\left(\mathscr{H}_{k}\right)$ содержится в $C P n_{*}(\mathscr{F})$. Для доказательства обратного включения прежде всего отметим, что любой элемент $G \in \mathbb{B}_{*}(\mathscr{F})$ представляет собой полуторалинейную форму, так что сушествуют полунормы $\|\cdot\|_{k}$ и $\|\cdot\|_{m}$, а также положительная постоянная $M$, зависящая от $G$, такие, что

$$
|G[u, v]| \leqslant M\|u\|_{k} \cdot\|v\|_{m} \quad \text { для } \quad u, v \in \mathscr{F}
$$

(см. доказательство такого же свойства для совместно непрерывных билинейных форм из $[10$, гл. 41, с. 420$])$. Следуя $(11)$, можно предположить, что $k=m$. Следовательно, $G$ имеет единственное совместно непрерывное расширение на $\mathscr{H}_{k}$, так как $\mathscr{F}$ плотно в $\mathscr{H}_{k}$. Пусть $\Phi \in C P n_{*}(\mathscr{F})$. Тогда $\Phi(X) \in \mathbb{B}_{*}(\mathscr{F})$ при каждом $X \in \mathscr{B}(\mathscr{H})$, и существует гильбертово пространство $\mathscr{H}_{k}$, в котором $\Phi(X)$ может быть расширено до совместно непрерывной полуторалинейной формы. Обозначим это расширение тоже символом $\Phi(X)$. Индекс $k$ зависит от $\Phi$ и в общем случае от $X$, но мы будем считать, что можно выбрать один и тот же индекс $k$ для всех $X$, зависящий только от $\Phi$. В самом деле, рассмотрим гильбертово пространство $\mathscr{H}_{k}$, на которое можно непрерывным образом расширить $\Phi(I)$. Тогда согласно (4) имеем следующие неравенства для произвольного самосопряженного $X$, всех $\psi \in \mathscr{F}$ и некоторой положительной постоянной $M$ :

$$
|\Phi(X)[\psi]| \leqslant\|X\| \cdot|\Phi(I)[\psi]| \leqslant\|X\| M\|\psi\|_{k}^{2}
$$


Так как любой ограниченньй оператор можно представить в виде линейной комбинации самосопряженных, а отображение $\Phi$ линейно по операторной переменной, то $\Phi(X) \in \mathbb{B}_{*}\left(\mathscr{H}_{k}\right)$ для любого $X \in \mathscr{B}(\mathscr{H})$. Другими словами, $\Phi \in C P n_{*}\left(\mathscr{H}_{k}\right)$. Таким образом, получено (13).

Из (12) и (13) видно, что множество $C P n_{*}(\mathscr{F})$ может быть естественным образом снабжено топологией индуктивного предела. Согласно определению данная топология является наиболее сильной в $C P n_{*}(\mathscr{F})$, что делает непрерьвным отображение, осуществляющее вложение $C P n_{*}\left(\mathscr{H}_{k}\right)$ в $C P n_{*}(\mathscr{F}), k \geqslant 0$. Согласно предложению 1 топология, индуцированная $\mathscr{R} \mathscr{F}$, также делает непрерывными отображения вложения. Таким образом, эта топология слабее (или равна) топологии индуктивного предела. Естественньм образом возникает задача о возможной эквивалентности обеих топологий. Мы отсылаем к работе [11] за определением индуктивного предела в случае общих топологических пространств и его основными свойствами.

СледСТВИЕ 2. 1) Для любого $\Phi \in C P n_{*}(\mathscr{F})$ существуют плотно определенный положительный самосопряженный оператор $\Lambda$ и последовательность $\left\{A_{k}\right\}$ ограниченных операторов в $\mathscr{H}$ такие, что $\sum_{k} A_{k}^{*} A_{k}$ сильно сходится и выполнено (10).

2) Свойства а)-г) являются достаточными условиями того, ито $\Phi \in C P n_{*}(\mathscr{F})$.

3) Любой элемент из $C P n_{*}(\mathscr{F})$ является пределом последовательности әлементов из $C P n(\mathscr{H})$ относительно топологии, индуцированной $\mathscr{R} \mathscr{F}$.

ДоКАЗАТЕЛЬСТВО. Напомним, что $\mathscr{H}_{0}$ рассматривается в качестве исходного гильбертова пространства $\mathscr{H}$. Пусть $\Phi \in C P n_{*}(\mathscr{F})$. По теореме 2 существует индекс $k$ такой, что $\Phi \in C P n_{*}\left(\mathscr{H}_{k}\right)$. Для доказательства применим предложение 2 и следствие 1 к гильбертову пространству $\mathscr{H}_{k}$. А именно, пусть $\Lambda$ - плотно определенный положительный самосопряженньй оператор, удовлетворяющий условиям $(7) ;\left\{A_{k}\right\}$ - последовательность в $\mathscr{B}(\mathscr{H})$, а $\left\{R_{1 / k}\right\}$ - последовательность в $C P n(\mathscr{H})$, описанная в следствии 1. Последовательность $\left\{R_{1 / k}\right\}$ сходится к $\Phi$ в топологии $C P n_{*}\left(\mathscr{H}_{k}\right)$, но $C P n_{*}\left(\mathscr{H}_{k}\right)$ непрерьвно вложено в $C P n_{*}(\mathscr{F})$ (см. предложение 1$)$; следовательно, сходимость последовательности $\left\{R_{1 / k}\right\}$ имеет место и в топологии $C P n_{*}(\mathscr{F})$. Следствие доказано.

\section{4. Примеры и замечания.}

ПримеР 1. Приведем достаточное условие того, что отображение $\Phi \in C P n_{*}(\mathscr{F})$.

ПРЕДЛОЖЕНИЕ 4. Пусть $\Phi$ переводит $\mathscr{B}(\mathscr{H})$ в множество плотно определенных полуторалинейных форм на Н̈. Предположим, что

1) $\Phi(I)$ - плотно определенная замкнутая квадратичная форма на $\mathscr{H}$;

2) для любого $X \in \mathscr{B}(\mathscr{H})$ имеет место включение $\operatorname{dom} \Phi(I) \subseteq \operatorname{dom} \Phi(X)$;

3) $\Phi$ удовлетворяет условиям а), б) и г), а векторные переменные принадлеxam dom $\Phi(I)$.

Тогда $\Phi \in C P n_{*}(\mathscr{F})$ для любого банахова пространства $\mathscr{F}$ такого, что

$$
\mathscr{F} \subseteq \operatorname{dom} \Phi(I), \quad\|u\|_{\mathscr{F}}^{2} \geqslant a[u],
$$

$2 \partial e$

$$
a[u]=\|u\|_{\mathscr{H}}^{2}+\Phi(I)[u],
$$

и $\mathscr{F}$ плотно в $\operatorname{dom} \Phi(I)$ относительно нормы $a[u]^{1 / 2}$. 
ДокАЗАТЕльство. Так как $\Phi(I)$ замкнуто, то квадратичная форма, определенная на dom $\Phi(I)$ формулой (15) позволяет рассматривать данное пространство как гильбертово пространство со скалярным произведением $(u, v)_{\Phi}=(u, v)+\left(\Phi(I)^{1 / 2} u, \Phi(I)^{1 / 2} v\right)$, которое плотно и непрерывно вложено в $\mathscr{H}$. Пусть $\mathscr{H}_{1}=\operatorname{dom} \Phi(I)$. По предположению $\Phi$ удовлетворяет условиям а), б) и г). Осталось доказать условие в).

Как уже отмечалось, условия а) и б) влекут неравенство (4) для самосопряженных элементов из $\mathscr{B}(\mathscr{H})$. Таким образом, для любого самосопряженного элемента $X \in \mathscr{B}(\mathscr{H})$ $\Phi(X)$ является ограниченной полуторалинейной формой на $\mathscr{H}_{1}$. Так как произвольный элемент из $\mathscr{B}(\mathscr{H})$ представим в виде линейной комбинации самосопряженных операторов, мы приходим к тому же заключению для любого $X \in \mathscr{B}(\mathscr{H})$. Следовательно, условие в) вьполнено. Согласно следствию $1 \Phi \in C P n_{*}\left(\mathscr{H}_{k}\right)$. Если $\mathscr{F}$ - банахово плотное в $\mathscr{H}_{1}$ пространство такое, что справедливо $(14)$, то предложение 1 влечет $\Phi \in C P n_{*}(\mathscr{F})$.

Два следуюших примера связаны с незамыкаемыми квадратичными формами.

ПРИмеР 2. Рассмотрим отображение $\Phi \in C P n_{*}(\mathscr{F})$, для которого $\Phi(I)$ является плотно определенной замкнутой квадратичной формой на $\mathscr{H}$, хотя существует элемент $X \in \mathscr{B}(\mathscr{H})$ такой, что квадратичная форма $\Phi(X)$ незамькаема, несмотря на справедливость неравенства (4), и следовательно, не имеет операторной реализации.

Напомним, что квадратичная форма является замыкаемой, если она имеет замкнутое расширение [9, гл. VI, разд. 1]. Эквивалентным условием замыкаемости является следующее $[9$, теорема VI.1.17, с. 315]:

$$
\psi_{n} \in \operatorname{dom} a, \quad \psi_{n} \rightarrow 0 \text { и } a\left[\psi_{n}-\psi_{m}\right] \rightarrow 0 \text { при } n, m \rightarrow \infty \Longrightarrow a\left[\psi_{n}\right] \rightarrow 0 .
$$

Пусть $\mathscr{H}=\ell_{2}$ представляет собой пространство квадратично суммируемых последовательностей комплексных чисел. Пусть $N$ - оператор числа частиц, т.е.

$$
\operatorname{dom} N=\left\{\left(a_{n}\right) \in \mathscr{H}:\left(n a_{n}\right) \in \mathscr{H}\right\}, \quad N\left(\left(a_{n}\right)\right)=\left(n a_{n}\right) .
$$

Пусть $\mathscr{H}_{k}=\operatorname{dom} N^{k / 2}, k \in \mathbb{N} \cup\{0\},-$ пространство со скалярным произведением $\langle\varphi, \psi\rangle_{k}=\left\langle N^{k / 2} \varphi, N^{k / 2} \psi\right\rangle$.

Положим $\mathscr{F}=\bigcap_{k} \mathscr{H}_{k}$. Тогда $\mathscr{F}$ является пространством всех элементов из $\left\{a_{n}\right\} \in \mathscr{H}$ таких, что $\left\{n^{k} a_{n}\right\} \in \mathscr{H}$ для любого $k \in \mathbb{N} \cup\{0\}$. Известно, что $\mathscr{F}$ плотно в каждом $\mathscr{H}_{k}[12$, с. 142,143$]$. Положим

$$
\Phi(X)[\psi]=\left\langle N^{3 / 2} \psi, X N^{3 / 2} \psi\right\rangle
$$

Пусть $k=3, \Lambda=N^{3 / 2}, A_{1}=I$ и $A_{l}=0$ при $l \geqslant 2$. Для таких операторов $\Phi$ имеет структуру, описанную в следствии 1 . Это показьвает, что $\Phi \in C P n_{*}\left(\mathscr{H}_{3}\right)$ и, следовательно, $\Phi \in C P n_{*}(\mathscr{F})$. Более того, $\Phi(I)[\psi]=\left\|N^{3 / 2} \psi\right\|^{2}$ является замкнутой положительной плотно определенной квадратичной формой с $\operatorname{dom} \Phi(I)=\operatorname{dom} N^{3 / 2}$. Согласно неравенству Шварца (4) справедливо для любого $X \in \mathscr{B}(\mathscr{H})$. Пусть $\left\{e_{n}\right\}-$ канонический базис в $\mathscr{H}$, т.е. все координаты $e_{n}$ равны 0 за исключением единичной $n$-й компоненты.

Пусть $\psi_{n}=N^{-1 / 2} e_{n}=n^{-1 / 2} e_{n}$. Тогда $\psi_{n} \rightarrow 0$ и $N^{3 / 2} \psi_{n}=n e_{n}$. Пусть $x=$ $\{1 / n\}_{n=1}^{\infty} \in \mathscr{H}$ и $X=|x\rangle\langle x|$. Имеем

$$
\left\langle x, N^{3 / 2} \psi_{n}\right\rangle=\left\langle x, n e_{n}\right\rangle=1 .
$$


Для любого $\psi \in \mathscr{F}$

$$
\Phi(X)[\psi]=\left\langle N^{3 / 2} \psi, \mid x\right\rangle\left\langle x \mid N^{3 / 2} \psi\right\rangle=\left|\left\langle x, N^{3 / 2} \psi\right\rangle\right|^{2} .
$$

В частности, используя (16) и (17), получаем

$$
\Phi(X)\left[\psi_{n}\right]=\left|\left\langle x, N^{3 / 2} \psi_{n}\right\rangle\right|^{2}=1
$$

для всех $n . \mathrm{C}$ другой стороны,

$$
\Phi(X)\left[\psi_{n}-\psi_{m}\right]=\left|\left\langle x, N^{3 / 2}\left(\psi_{n}-\psi_{m}\right)\right\rangle\right|^{2}=0 .
$$

Это показывает, что $\Phi(X)$ является положительной плотно определенной, но незамыкаемой квадратичной формой.

ПРимеР 3. Рассмотрим некоторую модификацию примера 2 , когда $\Phi \in C P n_{*}(\mathscr{F})$ такой, что $\Phi(I)$ - незамыкаемая квадратичная форма на $\mathscr{H}$. Мы делаем упор на ключевой момент: $\Phi(I)$ необязательно должна быть замыкаемой квадратичной формой в исходном пространстве, но она обязана быть полуторалинейной формой в некотором гильбертовом пространстве, вложсенном в исходное плотным $u$ непрерывным образом.

Пусть $\mathscr{H}, \mathscr{F}$ и $x \in \mathscr{H}$ такие же, что и в примере 2. Пусть $y=x /\|x\|$. Тогда $|y\rangle\langle y|$ является ортогональным проектором. Определим $\Phi(\cdot)[\cdot, \cdot]$ следующим образом:

$$
\Phi(X)[u, v]=\langle\mid y\rangle\left\langle y\left|N^{3 / 2} u, X\right| y\right\rangle\left\langle y \mid N^{3 / 2} v\right\rangle .
$$

По-прежнему, $\Phi \in C P n_{*}(\mathscr{F})$, так как $\Phi \in C P n_{*}\left(\mathscr{H}_{3}\right)$ с $\Lambda=N^{3 / 2}, A_{1}=|y\rangle\langle y|$ и $A_{k}=0$ при $k \geqslant 2$. Далее,

$$
\Phi(I)[u]=\left|\left\langle y, N^{3 / 2} u\right\rangle\right|^{2} .
$$

Таким образом, квадратичная форма оказывается равной числу, умноженному на незамыкаемую квадратичную форму из примера 2 ; следовательно, она незамыкаема в $\mathscr{H}$.

ПримеР 4. Рассмотрим классическую гельфандовскую тройку $\mathscr{S} \subset \mathscr{H} \subset \mathscr{S}^{\prime}$, где $\mathscr{S}$ - пространство Шварца быстро убывающих гладких функций на $\mathbb{R}, \mathscr{H}$ - пространство квадратично интегрируемых по Лебегу функций, а $\mathscr{S}^{\prime}-$ пространство обобщенных функций ограниченного роста. Известно, что $\mathscr{F}$ является проективным пределом $\mathscr{H}_{k}-$ области определения квадратного корня из оператора гармонического осциллятора $N$, определенного формулой (5) (см. [8, с. 16]). При максимальной области определения в $\mathscr{H} N$ становится положительньм самосопряженным оператором.

Согласно теореме 2 и следствию 2 для любого $\Phi \in C P n_{*}(\mathscr{F})$ существует $k \in \mathbb{N} \cup\{0\}$ такое, что $\Phi \in C P n_{*}\left(\mathscr{H}_{k}\right)$. Таким образом, можно построить элементы $\Phi \in C P n_{*}(\mathscr{F})$ такие, что $\Phi(I)$ будет сингулярной квадратичной формой, аналогичной дельта-функции на $\mathbb{R}^{2}$, т.е. $\Phi(I)[u, v]=\bar{u}(0) v(0)$, где черта означает комплексное сопряжение.

Автор выражает благодарность профессору А. М. Чеботареву и профессору Р. Keсаде за интерес, проявленный к работе, и полезные замечания. 


\section{СПИСОК ЦИТИРОВАННОЙ ЛИТЕРАТУРЫ}

[1] Чеботарев А. М. О достаточных условиях консервативности минимальной динамической полугруппы // Матем. заметки. 1992. Т. 52. № 4. С. 112-127.

[2] Chebotarev A. M. Minimal solutions in classical and quantum probability // Quantum Probability and Related Topics. VII / ed. L. Accardi. Singapore: World Sci. Publ., 1992. P. 79-91.

[3] Chebotarev A. M., García J., Quezada R. A priori estimates and existence theorems for the Lindblad equation with unbounded, time-dependent coefficients // Recent Trends in Infinite Dimensional Non-Commutative Analysis. Publ. Res. Inst. Math. Sci. Kokyuroku. V. 1035. Kyoto: Kyoto Univ., 1998. P. 44-65.

[4] Parthasaraty K. R. An Introduction to Quantum Stochastic Calculus. Berlin: Birkhäuser, 1992.

[5] Bratelli O., Robinson D. W. Operator Algebras and Quantum Statistical Mechanics. I. Berlin: Springer, 1981.

[6] Kraus K. General state changes in quantum theory // Ann. Physics. 1971. V. 64. P. 311-335.

[7] Kelley J. L. General Topology. Grad. Texts in Math. New York: Springer, 1955.

[8] Obata N. White Noise Calculus and Fock Space. Lecture Notes in Math. V. 1577. Berlin-Heidelberg: Springer, 1994.

[9] Kato T. Perturbation Theory of Linear Operators. New York: Springer, 1966.

[10] Treves F. Topological Vector Spaces, Distributions and Kernels. New York-San Francisco-London: Acad. Press, 1967.

[11] Joshi K. D. Introduction to General Topology. New York-Chichester-Brisbane-Ontario-Singapore: Wiley, 1983.

[12] Reed M., Barry S. Methods of Modern Mathematical Physics. V. 1. Functional Analysis. New York-London-Toronto-Sidney-San Francisco: Acad. Press, 1980.

UAM-Iztapalapa, Depto. de Matemáticas, México

Поступило

E-mail : jcgc@xanum.uam.mx

30.12 .97 